\title{
A new algorithm for the downscaling of cloud fields
}

\author{
Victor Venema, ${ }^{\text {a }}$ Sebastián Gimeno García ${ }^{\mathrm{b}}$ and Clemens Simmer ${ }^{\mathrm{a}}$ \\ ${ }^{a}$ Meteorologisches Institut, Universität Bonn, Bonn, Germany \\ ${ }^{\mathrm{b}}$ Remote Sensing Technology, Technische Universität München, Munich, Germany \\ ${ }^{\star}$ Correspondence to: Victor Venema, Meteorologisches Institut, Universität Bonn, Auf dem Hügel 20, 53121 Bonn, \\ Germany. E-mail: victor.venema@uni-bonn.de
}

We present a novel algorithm for the downscaling of three-dimensional cloud fields. The goal of the algorithm is to add realistic subscale variability to a coarse field taking the resolved variability into account. The method is tested by coarse graining high-resolution sparse cumulus and broken stratocumulus clouds in the horizontal plane, downscaling these coarse fields back to the high resolution and comparing the radiative and microphysical properties of these downscaled fields with the original high-resolution fields. The resolutions of the cumulus and stratocumulus clouds used for this purpose are increased by a factor of four and ten, respectively. The downscaling decreases the errors in the flux transmittance and reflectance of the cumulus and stratocumulus cloud fields by at least a factor of ten and three, respectively, compared to utilising the coarse cloud fields. A novel aspect of our algorithm is the fact that it constrains the high-resolution fields of cloud liquid water content as well as the subscale cloud fraction. An alternative version that does not include cloud fraction information is less accurate, but still significantly better than using the coarse fields. The latter downscaling algorithm can also be utilised for the disaggregation of geophysical fields for which fractional coverages are not defined. Furthermore, the downscaling algorithm can be combined with our other algorithms to generate surrogate fields with other constraints, for example, surrogate clouds with a prescribed liquid water content height distribution. Copyright (C) 2010 Royal Meteorological Society

\footnotetext{
Key Words: disaggregation; stochastic modelling; 3D radiative transfer

Received 21 February 2009; Revised 20 August 2009; Accepted 7 October 2009; Published online in Wiley InterScience 15 January 2010
}

Citation: Venema V, Garcia SG, Simmer C. 2010. A new algorithm for the downscaling of cloud fields. Q. J. R. Meteorol. Soc. 136: 91-106. DOI:10.1002/qj.535

\section{Introduction}

The spatial resolution of cloud fields from dynamical atmospheric models is often insufficient to capture all scales relevant for cloud-radiation interactions and thus to serve as input to three-dimensional (3D) radiative transfer calculations (Koren et al., 2008). This is the case, for example, for numerical weather prediction (NWP) models, but also for many cloud resolving models (CRMs). To address this problem, we have developed a downscaling algorithm that produces 3D cloud fields with a higher resolution from such available coarse fields. The algorithm takes as input the coarse resolution fields of the mean liquid water content (LWC) and the cloud fraction.

Two statistical properties are essential for radiative transfer (RT) simulations. First of all, the distribution of the cloud water or optical thickness is important for taking into account the nonlinearity of RT. The assumption of no significant subscale variability, implicitly made by using coarse-resolution clouds, has similar consequences as the plane-parallel homogeneity assumption, i.e. the assumption that there is no variability on any scale. Both cases lead to 
biases in the radiation fields, e.g. producing too-bright cloud tops (Cahalan et al., 1994). Second, the structure of the cloud field, in the sense of two-point statistics, e.g. the spatial correlations, is also important for RT. Coarse-resolution clouds will tend to have longer correlation lengths than higher-resolution clouds. Both horizontally uncorrelated fields (Venema et al., 2006a) and fields with a correlation length much larger than the cloud depth (Chambers et al., 1997; Davis et al., 1997) have a higher reflectance than clouds with a correlation length in the order of the cloud depth. In the latter case, photons will scatter preferentially towards regions with lower extinction, which increases the transparency of clouds. This horizontal photon transport is especially important near cloud edges.

From these considerations, one can deduce that a downscaling algorithm aiming to improve RT should add subscale variability to the coarse field of the right amount and with the right spatial correlations. In our algorithm, both the variance and the spatial correlation of the subscale variability are estimated by extrapolating the power (variance) spectrum of the coarse cloud field. The algorithm is able to handle any extrapolated power spectrum. The code is similarly organized as the Iterative Amplitude Adjusted Fourier Transform (IAAFT) algorithm used for the stochastic generation of surrogate fields (Venema et al., 2006a, 2006c; Schmidt et al., 2007) and can easily be combined with it. The downscaling algorithm iteratively adjusts the spectrum and the coarse fields. Because of its iterative character, the algorithm is able to add the subscale variability while taking resolved small-scale features into account. For example, if the coarse field elements to the north are cloudy and to the south cloud-free, the algorithm will automatically insert more subscale cloudy pixels in the northern part. Furthermore, the algorithm is able to avoid negative LWC values.

Next to downscaling 3D NWP cloud fields, a 2D version of the algorithm could perform a downscaling of satelliteretrieved $2 \mathrm{D}$ liquid water path fields. A version without using information about the subscale cloud fraction could be used for the downscaling of measured or modelled precipitation and radiation fields. Such an algorithm for $2 \mathrm{D}$ radar-derived rain fields would be similar to the one of Ferraris et al. (2003) or Perica and Foufoula-Georgiou (1996). Their algorithms add multiplicative noise independently of the resolved field, leaving the coarse field visible in the final product. Many other downscaling algorithms have been developed; most perform, however, their downscaling on 1D time series (e.g. Olsson, 1998; Basu et al., 2004; Marani and Zanetti, 2007) or assume multifractal behaviour (e.g. Olsson, 1998; Basu et al., 2004). Perica and Foufoula-Georgiou (1996) find that adding noise independent of the smallest resolved scale can lead to deviations in the spatial correlations of their rain fields. This may be a reason why Olsson (1998) explicitly makes his perturbations a function of the resolved precipitation beforehand and afterwards.

\section{Data and methods}

\subsection{Methodology}

To quantify the quality of the downscaled fields, the downscaling algorithm is applied to coarsened large-eddy simulation (LES) clouds. From high-resolution 3D cloud fields, we calculate a coarse mean LWC field and a coarse cloud-fraction field. Based on these coarse fields the algorithm produces fields at the resolution of the original LES clouds. In this way, the physical and radiative properties of the downscaled surrogates can be accurately compared to the original high-resolution LES clouds.

We expect that the main applications of interest will be the downscaling of cloud fields from numerical weather prediction (NWP) and cloud resolving models (CRMs). Furthermore, a 2D version of the algorithm could be employed for the downscaling of satellite measurements. In both applications, not only is the mean cloud liquid water known, but also the subpixel cloud fraction. The algorithm will thus exploit information from both these fields.

We have chosen to coarsen the LES fields only horizontally, because NWP cloud fields are much better resolved vertically than horizontally. Furthermore, most of our LES cloud fields are relatively shallow. Consequently, after coarsening there would be no information left on the vertical LWC profile and its structure. For thicker clouds, it would be possible to adapt the algorithm to additionally perform the downscaling in the vertical. In this case, the vertical LWC profile that is subtracted before computing the statistics and added afterwards to the surrogate cloud, see subsection 3.1, would need to be interpolated. An elegant method for this would be a mean-preserving spline interpolation algorithm (Sheng and Zwiers, 1998).

\subsection{LES clouds}

The algorithm is validated on two sets of clouds: cumulus $(\mathrm{Cu})$ over land and stratocumulus $(\mathrm{Sc})$ over the ocean. The 51 cumulus fields represent a diurnal cycle and were generated in the framework of the Atmospheric Radiation Measurement (ARM) project (Brown et al., 2002) and are also employed and described in more detail in Venema et al. (2006a). The fields have a resolution of $100 \mathrm{~m}$ in the horizontal and $40 \mathrm{~m}$ in the vertical. The number of grid boxes is 66 by 66 horizontally with 122 height levels. For this study we use the layers between 1160 and $3040 \mathrm{~m}$. Additionally, two vertical slices in both directions are removed at the sides to obtain a cloud field with $64 \times 64$ pixels in the horizontal, which is coarsened to $16 \times 16$ pixels, i.e. the resolution is decreased fourfold to $400 \mathrm{~m}$.

The 29 stratocumulus fields originate from three model runs in which polluted marine stratocumulus clouds are dissolving (Chosson et al., 2007). The geometrical thickness and LWC of one of the model runs was validated against a measured case from the ACE-2 (Aerosol Characterisation Experiment) project; the others have similar initial conditions. The cloud field starts relatively homogeneous and slowly dissolves and organises itself into larger patches. The clouds have 35 layers with a vertical resolution of $10 \mathrm{~m}$, of which we use the layers between 685 and $1025 \mathrm{~m}$. The number of grid boxes is $200 \times 200$ pixels horizontally with a resolution of $50 \mathrm{~m}$. These fields are coarsened ten times to $20 \times 20$ pixels of $500 \mathrm{~m}$.

\subsection{Radiative transfer calculations}

The upward and downward fluxes are calculated using the Leipzig Monte Carlo Model (LMCM: Gimeno Garcia and Trautmann, 2003). The same model was used for 3D Monte 
Carlo (MC) and 1D independent pixel approximation (IPA) computations. The IPA takes the real variability in optical properties of the cloud fields into account, but does not allow for horizontal photon transport between the 1D columns.

The radiation calculations for all sets of clouds have been made for monochromatic solar radiation with a wavelength of $550 \mathrm{~nm}$ and with solar zenith angles (SZAs) of $0^{\circ}$ and $60^{\circ}$. The fluxes are computed for the upper and lower boundaries of the input cloud fields. The surface albedo is set to zero, periodic boundary conditions are applied and the number of photons amounts to $10^{7}$. The cloud fields are assumed to be in a vacuum for simplicity.

The cumulus clouds were assumed to have 300 drops per $\mathrm{cm}^{3}$, the stratocumulus clouds $200 \mathrm{~cm}^{-3}$. These droplet concentrations were used to relate liquid water content to the effective radius based on the expression of Peng and Lohmann (2003). A fixed number concentration fits better to reality than a fixed effective radius (Brenguier et al., 2000). The Slingo parametrization (Slingo et al., 1989) was utilised for calculating the optical properties - extinction coefficient, single scattering albedo and asymmetry parameter - from the microphysical parameters - the liquid water content and the number of droplets. The cloud scattering phase function was assumed to be the Henyey-Greenstein phase function.

\section{Algorithms}

The downscaling algorithm is iterative and has three iterative steps, as illustrated in Figure 1. The algorithm is initialised with a white-noise field. The first iterative step is the adjustment of the spectrum. Consecutively, the resulting coarse means are readjusted to the original coarse means. This second step produces jumps at the edges of the coarse pixels. Therefore, the third step of the iteration aims at removing these artefacts. Subsection 3.1 describes these steps in detail, after which subsection 3.2 details the extrapolation algorithm to extrapolate the power spectrum to small scales. The last subsection describes some additional fields that were generated to investigate the reasons for the performance of the algorithm.

\subsection{Basic downscaling algorithm}

Before the statistical input is computed (the power spectrum and the distribution), the algorithm computes the mean LWC height profile and subtracts it from the coarse mean field, i.e. the algorithm is run with LWC anomaly fields. This is done because correlations describe how variables change together, but not how their mean values relate to each other. It is therefore more elegant to subtract the mean profile and work with the power spectrum of the anomalies. After the iterative loop, the mean LWC profile is re-added to the surrogate field.

From the coarse anomaly field the Fourier spectrum is calculated and extrapolated down to smaller scales. The extrapolation algorithm used in this study is described in section 3.2. The downscaling algorithm can work with any spectrum; consequently any other extrapolation method can be used instead.

In the spectral adjustment step, the Fourier coefficients of a white-noise field (in the first iteration), or of the field after the third step of the previous iteration $\left(\mathbf{F}_{3}\right)$, are computed. The Fourier coefficients $\left(S_{k}\right)$ are complex numbers. In the spectral adjustment the magnitudes of the Fourier coefficients are replaced by those of the extrapolated spectrum $\left(E_{k}\right)$. The phases $\varphi_{k}=S_{k} /\left|S_{k}\right|$ remain unaltered. In this way, the variance at each scale (described by the Fourier magnitudes) is adjusted, but the position of the waves (described by the phases) is not changed. Thus, the new complex Fourier coefficients are given by $S_{k}^{\prime}=\left|E_{k}\right| \varphi_{k}$.

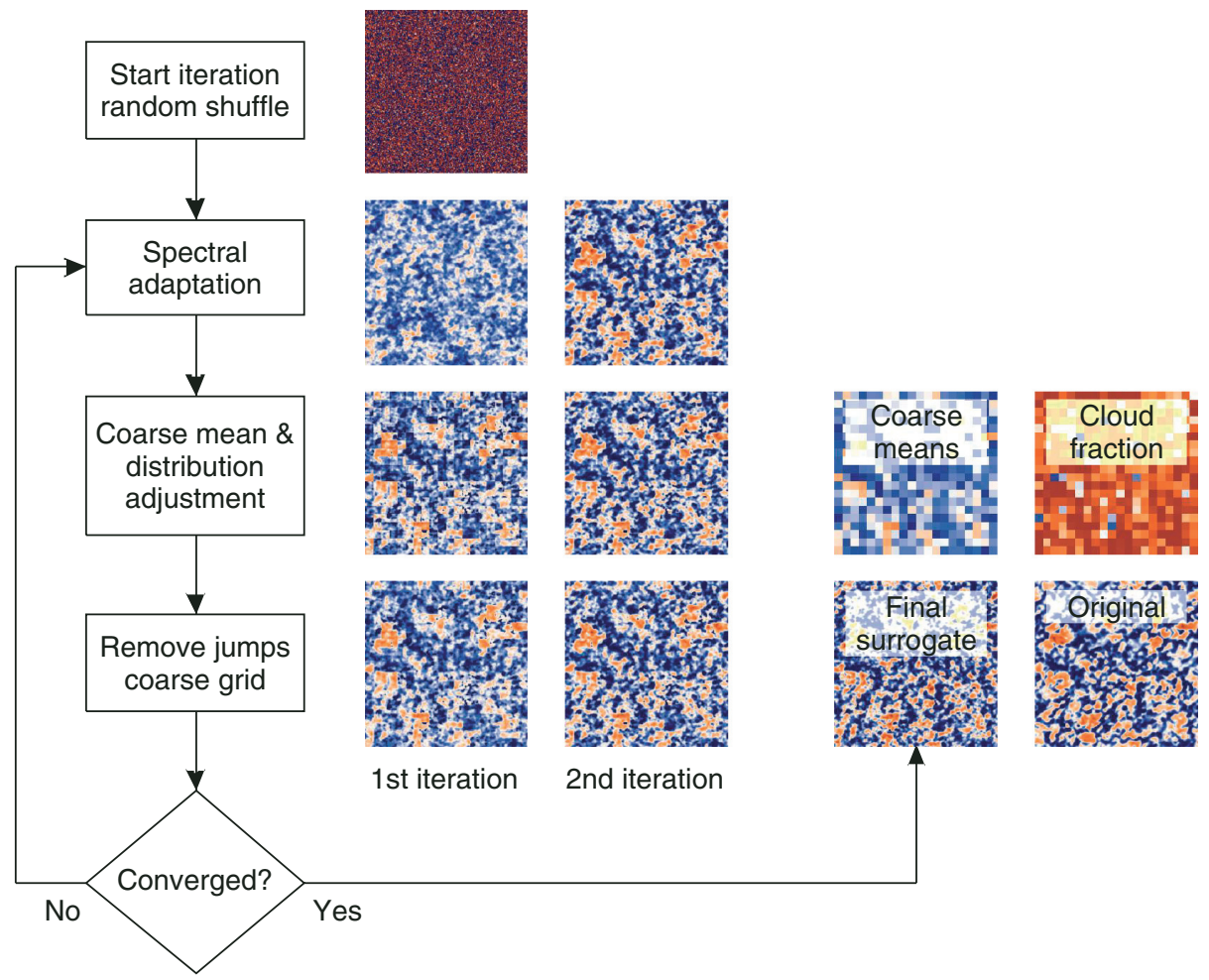

Figure 1. The flow chart of the downscaling algorithm. The result of the operations is illustrated by $2 \mathrm{D}$ horizontal cross-sections at the height of the maximum LWC, just below the top of this stratocumulus cloud. 
After an inverse Fourier transform of $S_{k}^{\prime}$, one obtains the field after the first iterative step, $\mathbf{F}_{\mathbf{1}}$.

In the second iterative step, the surrogate field is adjusted towards the coarse cloud fraction and coarse mean LWC fields. This step loops over all coarse pixels. For every coarse pixel, first the lowest values are set to an LWC of zero to match the coarse cloud-free fraction. In a second substep, a constant is added to every subpixel of the other fraction to make their mean equal to the prescribed cloudy fraction coarse mean. In a last substep, values that represent a negative LWC are set to an LWC of zero and we obtain field $\mathrm{F}_{2}$.

The second iterative step can create large jumps at the edges of the coarse pixels. These jumps are especially clear in the increment distribution of lag one, i.e. the difference in LWC between two adjacent pixels. This increment distribution has a fatter tail for increments that cross the coarse pixels than the increments in the middle of a coarse pixel. The too-strong jumps are partially removed by the spectral adjustment. The Fourier spectrum is equivalent to the second-order structure function (Davis et al., 1999). The structure function for a certain lag is given by the width of the increment distributions. Thus the spectrum can constrain the width of the increment distribution, but does not constrain its other moments. The spectral adjustment can therefore only adjust the width of the increment distribution, but not the fatness of the tails.

Therefore, the third iterative step adjusts the increment distribution of lag one over the coarse edges to the increment distribution in the middle of the coarse pixels. This adjustment is performed in the same way the normal IAAFT algorithm adjusts the distribution of the surrogate to the measured distribution, i.e. increments over a coarse edge are substituted with increments in the middle that have the same rank. The two LWC values that make up an increment are both changed by the same amount in opposite directions to obtain the new increment. This increment adjustment is performed once for increments over coarse edges in the east-west direction and once for increments in the north-south direction, after which we obtain field $\mathbf{F}_{3}$.

The aim of the above increment adjustment is to remove artefacts from the second iterative step. Because this adjustment is not a necessary requirement for the final surrogate fields, the main iterative loop is executed twice: the first time with the increment adjustment, the second time without. The convergence criterion for both loops is, just as in the stochastic IAAFT algorithm (SIAAFT: Venema et al., 2006b), the number of iterations without an improvement in accuracy of more than $1 \%$. The accuracy is determined as the difference of the Fourier spectrum of the final surrogate and the spectrum of the original cloud. For the cumulus clouds we set the threshold to 200 iterations without significant improvements; for stratocumulus to 20. It is expected that these thresholds are so high, that the number of iterations is not a limiting factor for the accuracy of the results.

For this study, we produced an ensemble of ten surrogate fields for every LES cloud and only used the surrogate with the best matching power spectrum. As the accuracy of the ensemble members did not differ much, the results would likely not be much different if only one surrogate had been generated, but for this study we aimed for a high accuracy.

\subsection{Extrapolated spectrum-standard downscaled cloud fields}

The downscaling algorithm can utilize any given power spectrum and is thus independent of the extrapolation algorithm used. To generate the cloud fields a spectrum in Cartesian coordinates $E_{k}=E_{k_{x}, k_{y}, k_{z}}$ is needed. However, the extrapolation is easier to explain in a polar $E_{k}=E_{k_{r}, k_{\theta}, k_{\varphi}}$ or cylindrical $E_{k}=E_{k_{r}, k_{\theta}, k_{z}}$ coordinate system, with the radius $r$, the angle in the vertical plane $\theta$, and the angle in the horizontal plane $\varphi$. A straightforward method would be to extrapolate the power spectrum by modelling the smallscale variance as a fractal power-law function $\left(E_{k} \sim|k|^{b}\right)$, where the spectral exponent would need to be a function of the elevation angle $\theta$ to account for the stability of the atmosphere and maybe of the azimuth $\varphi$ in case of horizontal anisotropies. This view is most appropriate if the cloud water is understood as a conservative passive additive to homogeneous turbulence (Tatarski, 1961; Erkelens et al., 2001). This assumption likely holds and is often found to be an accurate description for stratiform clouds over the ocean (Cahalan et al., 1994; Davis et al., 1999), but may fail for ice clouds or convective clouds. In the case of ice clouds, the large crystals may not be an additive that moves with the wind; Hogan and Kew (2005) have studied the complicated structure of ice clouds measured by radar. For convective clouds, the cloud water is neither conservative due to phase transitions, nor passive due to condensation and buoyancy; a non-fractal young cumulus field was, for example, measured in situ by Schmidt et al. (2007).

In general, the information on the spectrum at small scales may be obtained from higher-resolution models or measurements. For the downscaling of fields from NWP or CRM models, one may use LES clouds or higherresolved model runs over a smaller region in order to find a good way to model the small scales. For downscaling satellite measurements, ground-based or airborne imagers may provide the needed small-scale information.

The structure of the LES clouds used in this study can only be well approximated by a fractal power-law Fourier spectrum at small scales: relative to the power law at small scales, the spectrum is missing more and more variance towards the larger scales and is flat at scales comparable with the size of the model domain. In a double logarithmic plot a power law becomes a line. In such a plot the power spectrum is mostly flat at large scales and then slowly bends down to become a straight line at small scales. This nonfractal structure may be physical due to the abovementioned reasons, but may also well be an artefact of the periodic boundary conditions of the LES models. The latter condition does not allow for variance at larger scales and disallows large-scale vertical motion.

The small scales of the coarse field are in the bend where the double logarithmic plot is still becoming steeper. Because of this curvature of the spectrum, fitting a power law to the small scales of the coarse field would result in too much variance at small scales. Therefore, working in a cylindrical coordinate system, the horizontally isotopic power spectra (averaged over the azimuth angle $\varphi$ ) was computed for every wave number in the vertical $\left(k_{z}\right)$. These non-fractal isotropic spectra, including the bend described above, were then averaged over all clouds. The cumulus and stratocumulus clouds have their own empirical functions. These empirical functions were consecutively fitted to the 
power spectra of the coarse fields to compute the new highresolution spectral coefficients. The fit was computed by setting the variance (power) of the empirical function equal to the mean variance over a common range of scales given by the small scales of the coarse spectrum.

\subsection{Alternative downscaled cloud fields}

The downscaled cloud fields will be compared with the original high-resolution fields and with the coarse-grained fields; the abbreviations that will be used later on are indicated in italics. The LWC of the latter fields has a coarse structure, but the computational resolution is the same as the downscaled clouds for better comparison of the pixel scale noise levels. Linearly interpolated clouds were derived from the coarse-grained fields using periodic boundary conditions, which are only utilised for a comparison of the autocovariance functions (section 4.3).

Next to these fields, a number of variations on the downscaling algorithm were developed for a better understanding of the importance of the various steps of the algorithm. To investigate the importance of the spectral extrapolation algorithm, the standard surrogates with the extrapolated spectrum, will be compared to surrogates that were generated with the exact spectrum of the original LES clouds as input.

An alternative algorithm was developed that generates downscaled surrogates assuming that no cloud fraction information is available. This algorithm has an alternative formulation for the second iterative step, i.e. the adjustment of the coarse mean fields. This step starts by adjusting the coarse mean in the same way as the standard algorithm. Then the negative cloud water values are set to zero. The sum of the liquid water content that is added this way is computed. This sum is subtracted from the cloudy subpixels: starting with the subpixel with the smallest positive LWC value, the LWC is reduced to zero one by one (the last value is reduced to a positive value to exactly compensate for the added LWC sum).

To investigate the influence of the third iterative step, i.e. the increment adjustments at the coarse edges, surrogates are also generated without this step, marked as no edge reduction. To make this algorithm as similar to the standard algorithm as possible, it also executes the main iterative loop twice.

\section{Results}

\subsection{Liquid water content}

Results for four out of a total of eighty original cloud fields are displayed as examples in Figure 2. Two cumulus and two stratocumulus cloud examples are shown. As aimed for, the surrogate clouds display more variability than the coarse fields. The colour scale is chosen to highlight the differences for pixels with little LWC by making cloud-free pixels dark blue and the smallest LWC values white/silver. In this way one can see that the small LWC values of the surrogate cumulus clouds, especially in the leftmost column, are noisier than the original LES clouds, which leads to positive biases in the reflectance. The noise in the vertical leads to a bias in the cloud cover; depending on the LWP threshold chosen to distinguish cloudy from cloudfree columns, the cloud cover of the surrogates is a few to about $7 \%$ too high for the stratocumulus clouds and around $7 \%$ too high for the cumulus clouds. The cloud cover of the surrogates is too high although their cloud fraction (the fraction of $3 \mathrm{D}$ cloudy pixels) is too low by 2 to $3 \%$. The latter is caused by the last step of the coarse
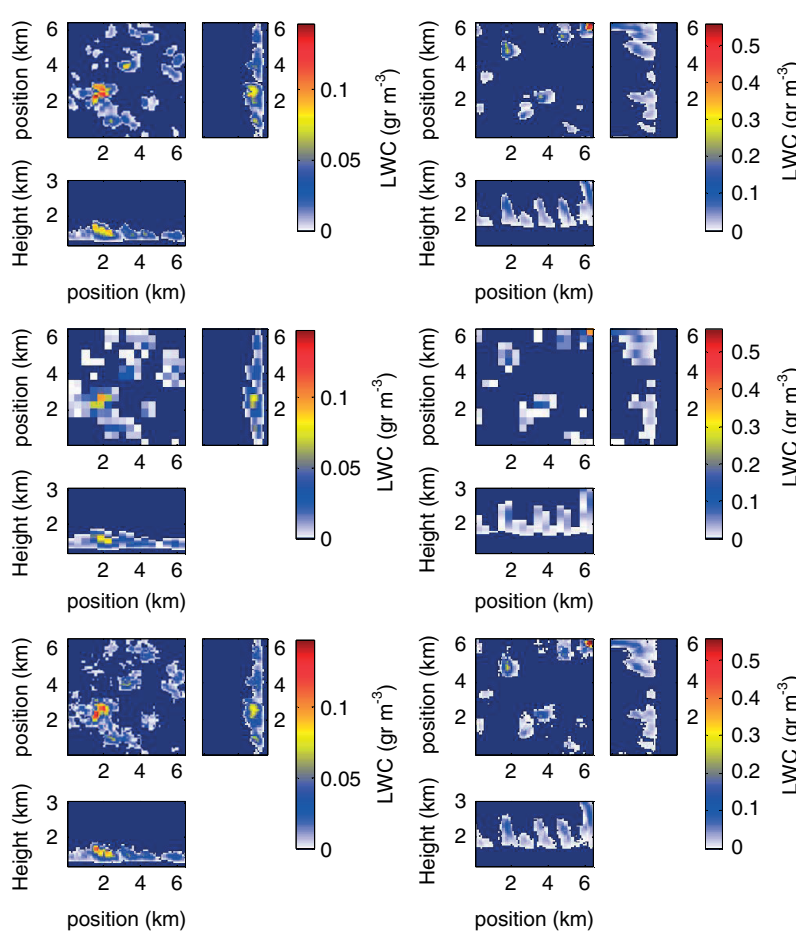
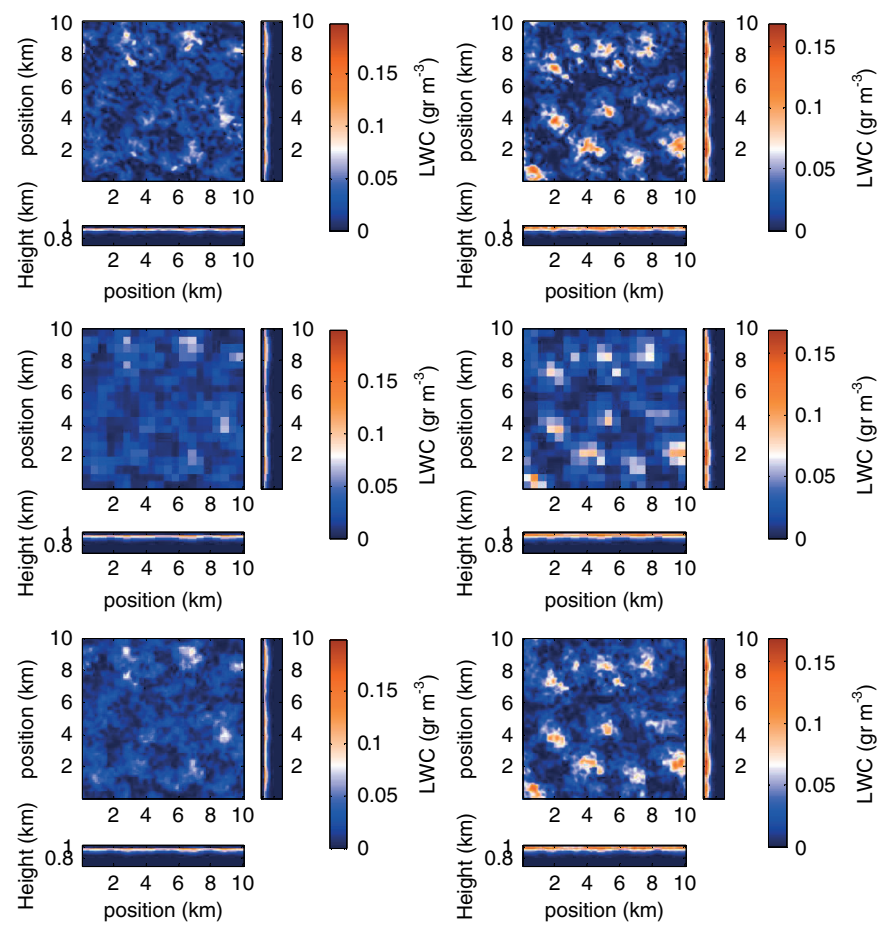

Figure 2. Examples of two cumulus (left two columns) and two stratocumulus (right two columns) LES clouds and their corresponding coarse-grained and surrogate fields. The first row depicts the original 3D LES clouds; the largest square subplot is the average LWC seen from the top, the bottom subplot is a front view and the right subplot a side view. The second row represents the coarse-grained cloud fields. The third row shows surrogates with an extrapolated spectrum. 
(a)

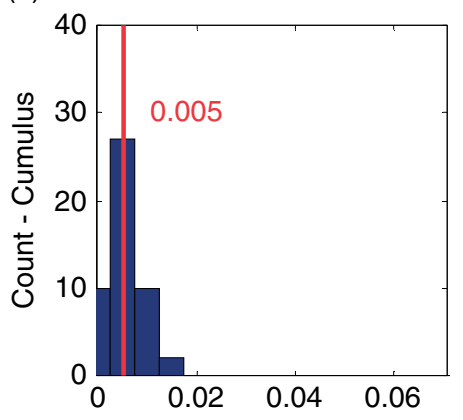

(d)

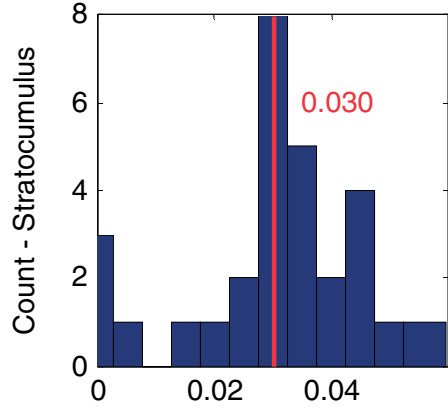

(g)

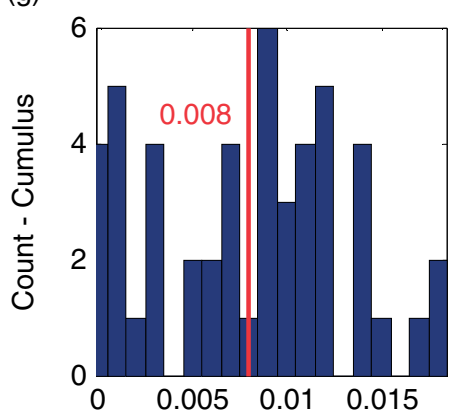

(j)

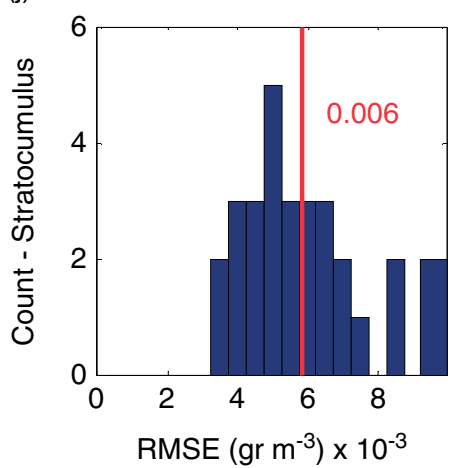

(b)

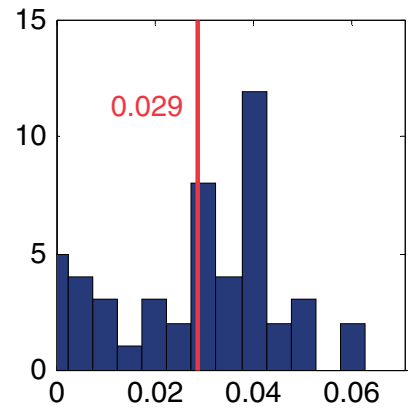

(e)

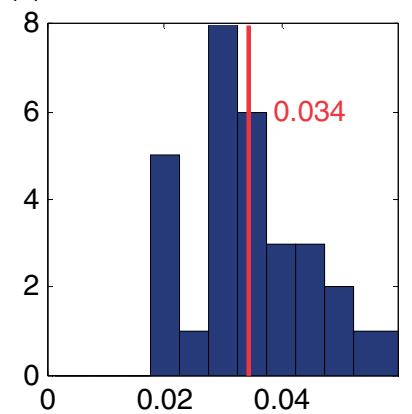

(h)

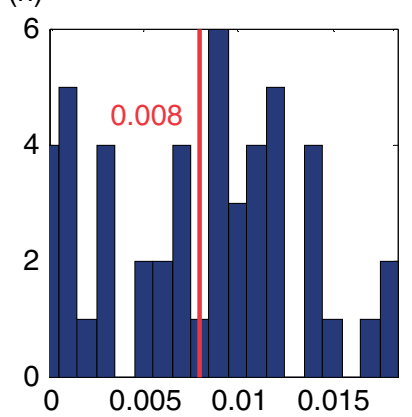

(k)

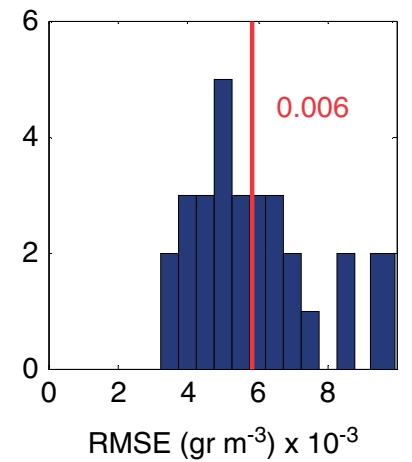

(c)

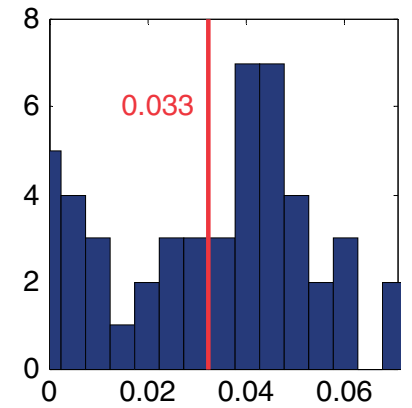

(f)

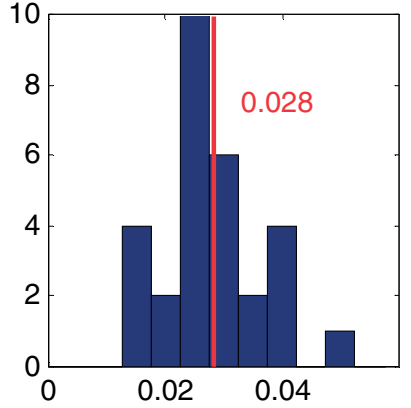

(i)

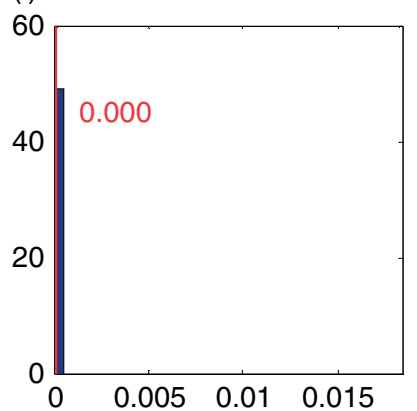

(I)

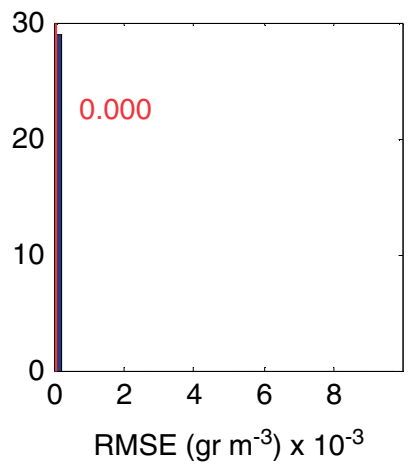

Figure 3. Histograms of the root-mean-square difference of the high-resolution ((a)-(f); top two rows) and coarse-resolution ((g)-(l); lower two rows) 3D liquid water content fields between the synthetic fields and the original clouds. The first and third rows display the cumulus clouds and the second and fourth rows present the stratocumulus clouds. The left column depicts the histogram for the surrogates with the perfect spectrum. The middle column displays the surrogate with an extrapolated spectrum. The right column represents the coarse-grained cloud fields. The red (or dark grey in the print version) line and number indicate the mean errors. This figure is available in colour online at www.interscience.wiley.com/journal/qj

field adjustment. In this step the cloud fraction is reduced if it is too high, but if it is too low no cloud-free pixels are made cloudy, the net effect of which is a bias towards a too-low cloud fraction. Looking carefully, the edges of the coarse cloud fields are still partially visible in the surrogate clouds.

The differences between the original cloud fields and the surrogates is shown quantitatively in Figure 3, which displays the histograms of the root-mean-square error (RMSE) of the LWC fields at the coarse- and the high-resolution scale. The histograms demonstrate that the high-resolution RMSE of the surrogate LWC fields do not show a clear improvement over the coarse fields. There is one exception: the surrogate cumulus clouds which are based on the exact spectrum of the original clouds do show a much lower RMSE. This is likely an artificial result; see discussion. 
(a)

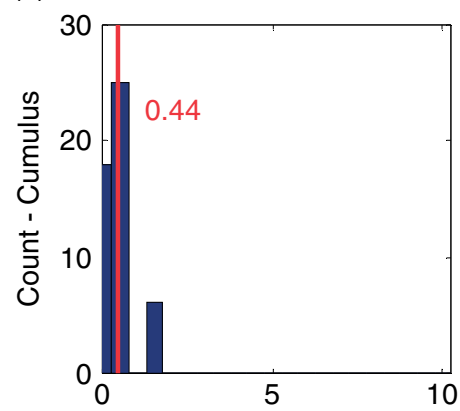

(d)

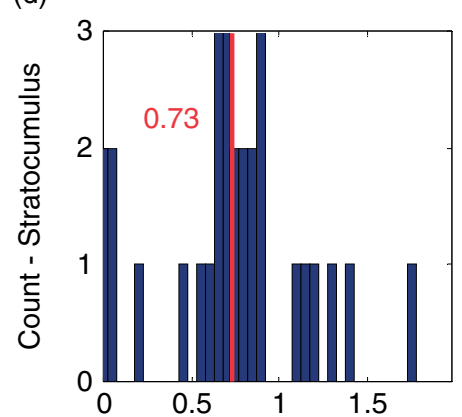

(g)

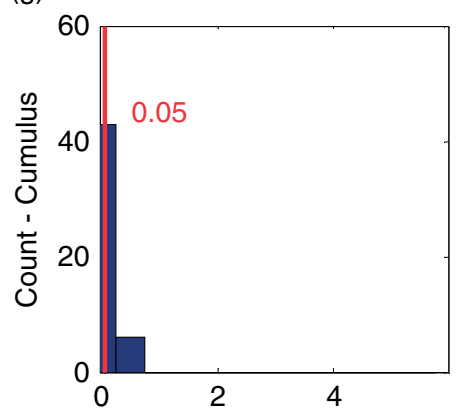

(j)

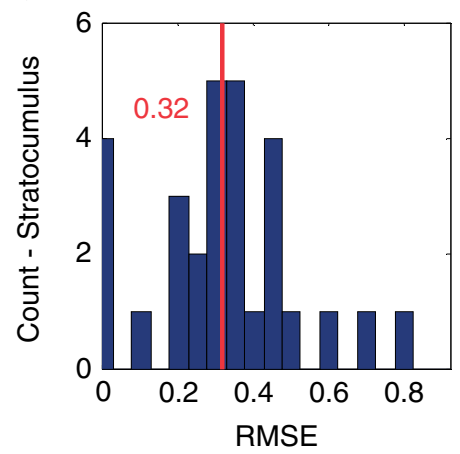

(b)

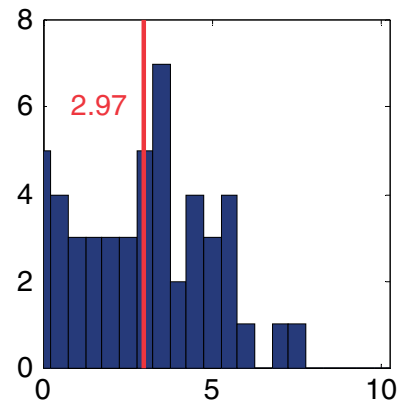

(e)

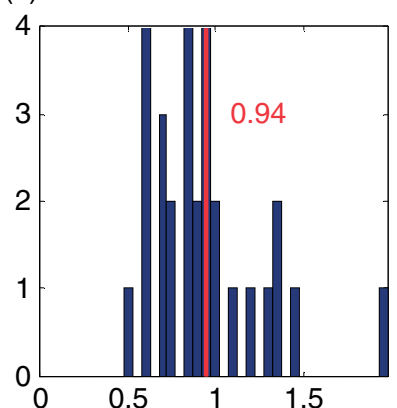

(h)

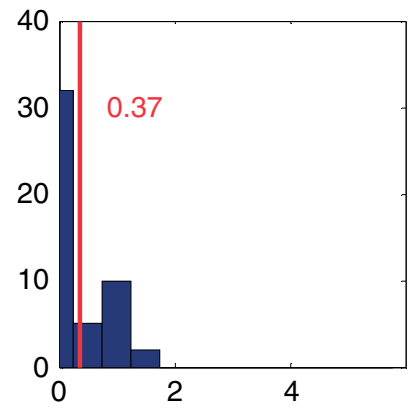

(k)

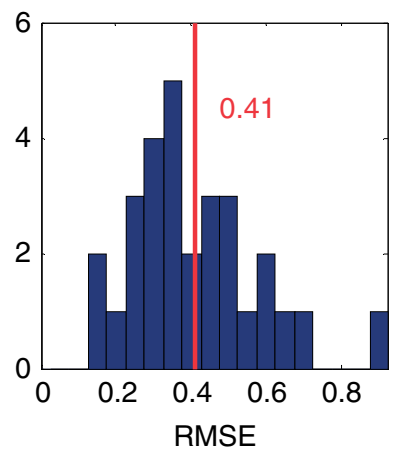

(c)

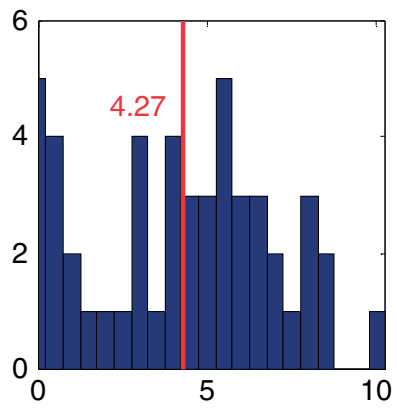

(f)

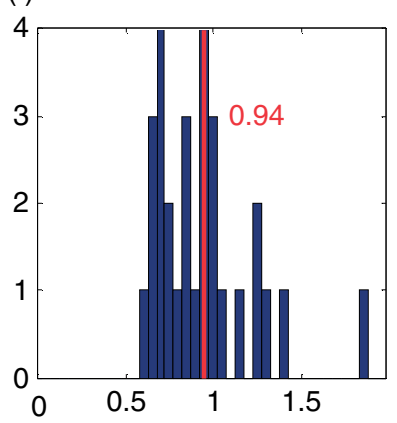

(i)

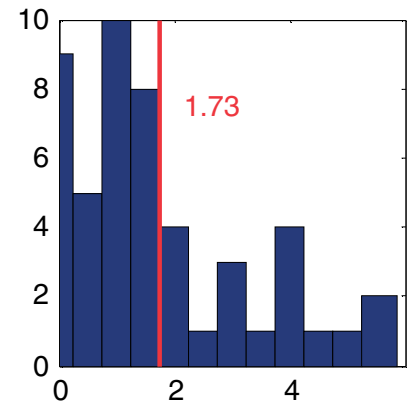

(I)

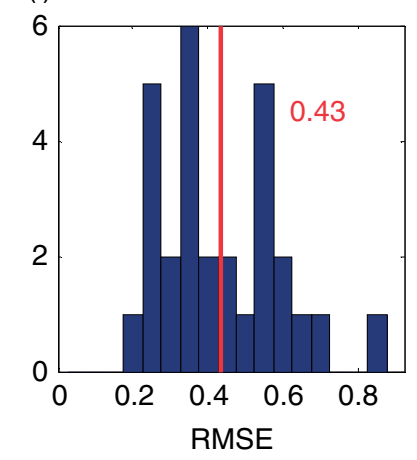

Figure 4. Histograms of the root-mean-square difference of the high-resolution ((a)-(f); top two rows) and coarse-grained ((g)-(l); lower two rows) $2 \mathrm{D}$ optical depth fields between the synthetic fields and the original clouds. The first and third rows are for the cumulus clouds, the second and fourth for the stratocumulus clouds. The first column is for the surrogate with an exact spectrum, the middle column for the surrogates with an extrapolated spectrum and the right column for the coarse-grained LES clouds. This figure is available in colour online at www.interscience.wiley.com/journal/qj

The RMSEs at the coarse scale are much smaller than on the high-resolution scale. The errors for the surrogate clouds are not zero because the adjustment of the coarse mean LWC is not the last step in the algorithm. The coarsescale errors of the coarse fields are zero, as they should be. Please note that the coarse-scale histograms of the two types of surrogate field are identical, but the errors themselves are not. The differences between the errors are, however, at least two orders of magnitude less than the errors themselves and do not influence the histograms.

\subsection{Optical depth}

Figure 4 shows the same histograms as Figure 3 for the optical depth. The high-resolution RMS errors of the surrogate clouds are not much different from the coarse fields for the stratocumulus fields. However, the cumulus clouds do show differences, especially those based on exact spectral information; see comments on this phenomenon for Figure 3.

At the coarse scale, the differences between Figures 3 and 4 are clear and point to the importance of the subscale 
Table I. Average error in optical depth over all LES cloud fields.

\begin{tabular}{|c|c|c|c|c|}
\hline Field & Overall bias fields & RMSE of field averages & RMSE at coarse resolution & RMSE at fine resolution \\
\hline \multicolumn{5}{|l|}{ Cumulus } \\
\hline Coarse field & 0.5111 & 0.6164 & 1.7281 & 4.2682 \\
\hline Extrapolated spectrum & 0.0174 & 0.0468 & 0.3653 & 2.9712 \\
\hline Exact spectrum & 0.0005 & 0.0081 & 0.0544 & 0.4364 \\
\hline No cloud cover & 0.1088 & 0.1447 & 0.6533 & 2.8003 \\
\hline $\begin{array}{l}\text { No increment adjustment } \\
\text { Stratocumulus }\end{array}$ & 0.0218 & 0.0514 & 0.3596 & 3.1846 \\
\hline Coarse field & 0.1733 & 0.1826 & 0.4343 & 0.9399 \\
\hline Extrapolated spectrum & 0.0451 & 0.0487 & 0.4074 & 0.9418 \\
\hline Exact spectrum & 0.0047 & 0.0082 & 0.3170 & 0.7349 \\
\hline No cloud cover & 0.0405 & 0.0502 & 0.3899 & 0.9301 \\
\hline No increment adjustment & 0.0453 & 0.0488 & 0.4070 & 0.9475 \\
\hline
\end{tabular}
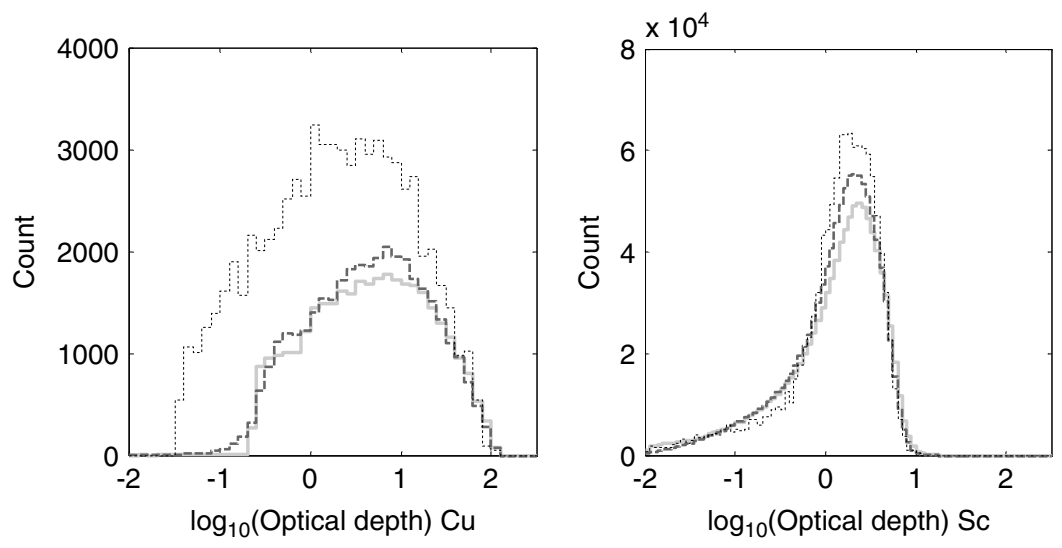

Figure 5. Histograms of the high-resolution optical depth distribution of the sparse cumulus clouds (left) and dissolving stratocumulus clouds (right). The original LES clouds are depicted with the thick light lines, the surrogates with extrapolated spectrum with a dashed line and the coarse fields with a thin black dashed line. Optical depth values below 0.01 (mainly zeros) are not shown, which explains the differences in the total number of counts.

variability. The coarse fields that have no error in the LWC do have considerable error with respect to the optical depth. The error of the surrogate cloud fields is smaller than the error of the coarse fields, especially for the cumulus clouds. The improvement of the surrogates over the coarse fields is very clear for the bias and the RMSE of the field mean optical depth, which are given in Table I. This table also lists all four error measures and also includes the surrogates without cloud fraction adjustment and without increment adjustment. The adjustment of the increment distribution over the coarse edges is found to have little influence on the errors of the surrogate optical depth fields. The surrogate cloud fields generated without cloud fraction information are less accurate for stratocumulus. For the cumulus clouds these surrogates are much less accurate, but still better than the coarse fields. For interpreting the absolute errors, please note that the mean optical depth is 1.8 for the sparse cumulus fields and 1.7 for the dissolving broken stratocumulus fields and that the standard deviations of the optical depth are 1.2 and 0.8 , respectively. The optical depth of the cumulus clouds is low due to their low cloud cover; the dissolving stratocumulus clouds have a very low LWC.

The improvement of the coarse cloud fields due to the downscaling can not only be expressed by the root-mean-square differences at various scales, but can also be seen in the optical depth distribution; see Figure 5. The most obvious difference is seen for the cumulus clouds where the optical depth distribution of the coarse fields contains many more small values than the original and surrogate fields. This also leads to a large difference in the total number of counts of the histograms, as the zero optical depth values are not shown due to the logarithmic abscissa. For stratocumulus clouds one can also observe that the distribution of the coarse fields is narrower than those of the original and surrogate fields. The surrogate downscaled cumulus and stratocumulus fields do have too many average optical depth values, but much less so than the coarse fields.

\subsection{Structure of the LWC}

The 1D autocovariance functions, shown in Figure 6, are computed in the horizontal plane over all possible rows in the east-west and north-south directions and averaged over all height levels and cloud fields. The figure clearly shows that the coarse-grained LWC fields and, even more, the linearly interpolated LWC fields lack small-scale variance. Defining the correlation length as the lag at which the covariance is $1 /$ e times the variance, the figure furthermore shows that these two fields are too smooth in the sense that their correlation length is too large. The surrogates with the exact spectrum have almost the same autocovariance function. The surrogates with the extrapolated spectrum 

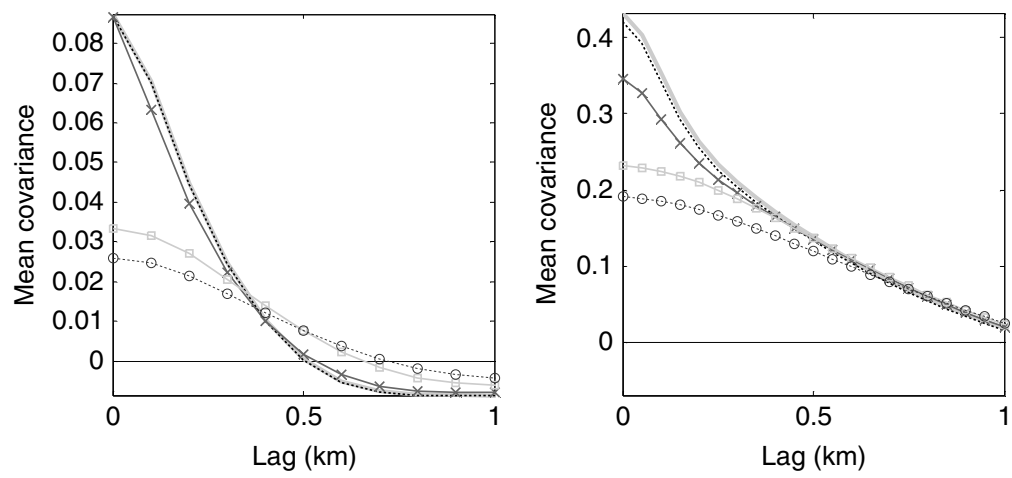

Figure 6. The autocovariance functions of the LWC fields. The left subplot shows the cumulus clouds and the right subplot the stratocumulus clouds. The thick light grey lines denote the original LES clouds, the thin dotted lines the surrogate with exact spectrum, the dark grey lines with crosses the surrogate with extrapolated spectrum, the light grey line with squares the coarse fields and the black dashed lines with circles the interpolated fields.
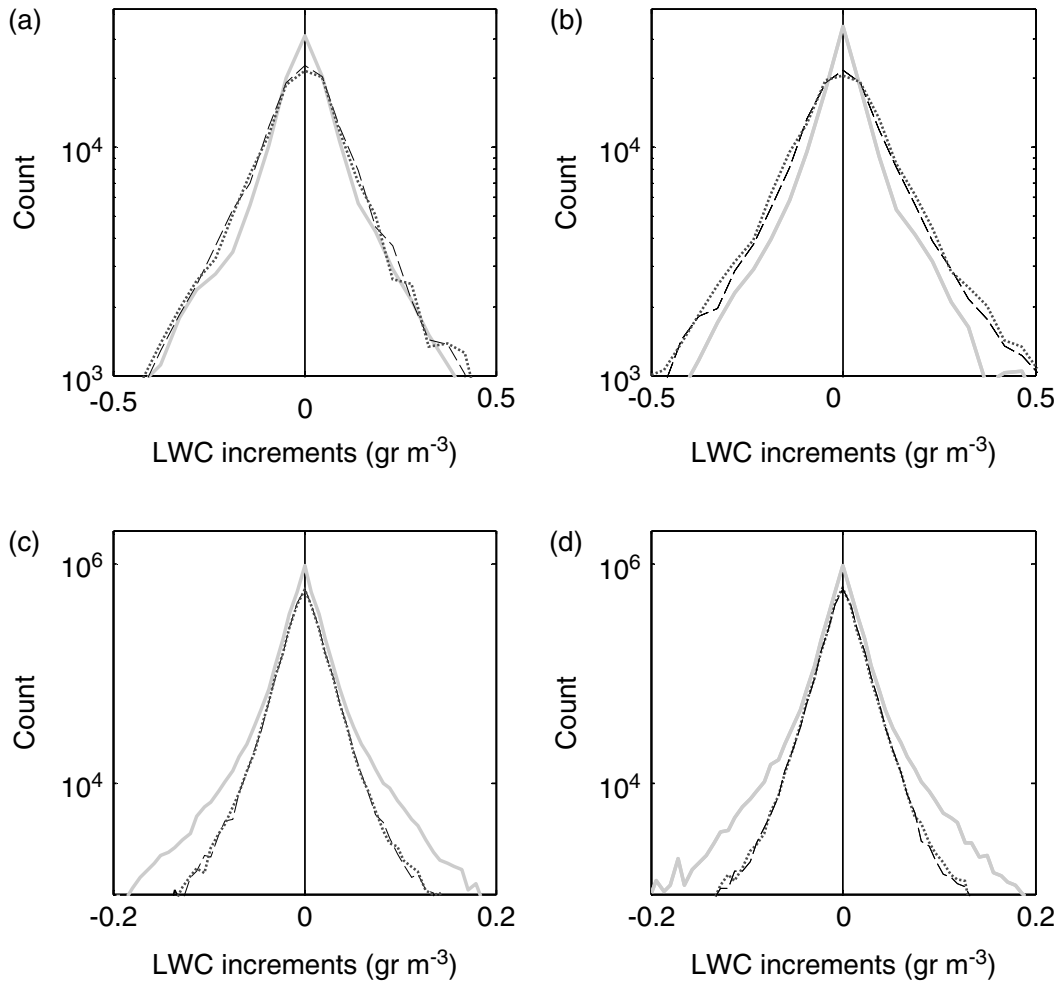

Figure 7. The increment distributions for horizontally neighbouring pixels of the cumulus (top row: (a), (b)) and stratocumulus (lower row: (c), (d)) clouds. The panels in the left column display the increment distributions calculated in the middle, i.e. without a coarse edge between the increments. The panels in the right column depict distributions of increments between pixels in adjacent coarse pixels. The grey thick lines represent the original LES clouds, the thin dashed lines the standard surrogates and the dotted lines the surrogate without the increment adjustment over the coarse edges.

underestimate the small-scale variance, especially the stratocumulus surrogates. The correlation length of the cumulus clouds is slightly underestimated; this length is, in turn, overestimated for the stratocumulus surrogates. The close match for the surrogates based on the exact spectrum and the deviations found for the surrogate with an extrapolated spectrum indicate that the cause of the latter deviations is the extrapolation algorithm and not the downscaling algorithm.

To investigate the increment distributions and the influence of the coarse edges on them, we have computed the increment histograms displayed in Figure 7. The increments in the middle of the coarse pixels match well for the cumulus fields (Figure 7(a)). However, the increment distribution of the stratocumulus surrogates (Figure 7(c)) has too-narrow tails; similar behaviour was found for a range of geophysical time series in Venema et al. (2006c): Surrogates tend to have too little variance of the variance (intermittence), probably as a result of the Fourier transform. In the original LES clouds, the increment distributions in the middle and at the edge of the coarse pixels are nearly identical; this distribution can thus be used to compare the distributions of the surrogates. It can be seen in Figure 7(b) that at the coarse edges the cumulus increments of the surrogates are larger than in the middle and too large compared to the originals. For the stratocumulus surrogates the coarse edges do not lead to a broader increment distribution; this distribution is, however, too narrow compared to the one of the original clouds, just as in the middle of the coarse pixels. From the difference between the surrogates with and without the increment adjustment one can observe that this step has a small, but positive, influence on the cumulus surrogates and no influence on the stratocumulus surrogate fields. 
Table II. Mean difference in mean reflectance and transmittance.

\begin{tabular}{|c|c|c|c|c|}
\hline \multirow[t]{2}{*}{ Field } & \multicolumn{2}{|c|}{ Cumulus } & \multicolumn{2}{|c|}{ Stratocumulus } \\
\hline & Refl. & Transm. & Refl. & Transm. \\
\hline \multicolumn{5}{|l|}{$S Z A=0^{\circ} 3 D R T$} \\
\hline Original & -0.000006 & 0.000006 & 0.000013 & -0.000013 \\
\hline Coarse field & 0.022002 & -0.022002 & 0.009565 & -0.009565 \\
\hline Extrapol. spectrum & 0.001765 & -0.001765 & 0.002555 & -0.002555 \\
\hline Exact spectrum & 0.000040 & -0.000040 & 0.000056 & -0.000056 \\
\hline No cloud cover & 0.005706 & -0.005706 & 0.002164 & -0.002164 \\
\hline No edge reduction & 0.001842 & -0.001842 & 0.002555 & -0.002555 \\
\hline \multicolumn{5}{|l|}{$S Z A=60^{\circ} 3 D R T$} \\
\hline Original & -0.000026 & 0.000026 & -0.000016 & 0.000016 \\
\hline Coarse field & 0.043428 & -0.043428 & 0.033831 & -0.033831 \\
\hline Extrapol. spectrum & 0.004431 & -0.004431 & 0.011788 & -0.011788 \\
\hline Exact spectrum & 0.000411 & -0.000411 & 0.003287 & -0.003287 \\
\hline No cloud cover & 0.011400 & -0.011400 & 0.010816 & -0.010816 \\
\hline No edge reduction & 0.005238 & -0.005238 & 0.011994 & -0.011994 \\
\hline \multicolumn{5}{|l|}{$S Z A=0^{\circ} I P A$} \\
\hline Original & 0.000007 & -0.000007 & 0.000001 & -0.000001 \\
\hline Coarse field & 0.022920 & -0.022920 & 0.009528 & -0.009528 \\
\hline Extrapol. spectrum & 0.002113 & -0.002113 & 0.002436 & -0.002436 \\
\hline Exact spectrum & 0.000028 & -0.000028 & -0.000005 & 0.000005 \\
\hline No cloud cover & 0.006175 & -0.006175 & 0.002204 & -0.002204 \\
\hline No edge reduction & 0.002458 & -0.002458 & 0.002415 & -0.002415 \\
\hline \multicolumn{5}{|l|}{$S Z A=60^{\circ} I P A$} \\
\hline Original & 0.000003 & -0.000003 & 0.000020 & -0.000020 \\
\hline Coarse field & 0.042657 & -0.042657 & 0.033627 & -0.033627 \\
\hline Extrapol. spectrum & 0.004147 & -0.004147 & 0.012023 & -0.012023 \\
\hline Exact spectrum & 0.000460 & -0.000460 & 0.003510 & -0.003510 \\
\hline No cloud cover & 0.011387 & -0.011387 & 0.011167 & -0.011167 \\
\hline No edge reduction & 0.004873 & -0.004873 & 0.012127 & -0.012127 \\
\hline
\end{tabular}

\subsection{Radiative fluxes}

To study the influence of the downscaling algorithm on the radiative properties of the clouds we have calculated their flux reflectance and transmittance with the help of a 3D Monte Carlo (MC) model. The mean transmittance for an SZA of $0^{\circ}$ of the surrogate and coarse fields is compared with the transmittance of the original clouds in Figure 8 Results for all types of synthetic fields, for both full 3D and $1 \mathrm{D}$ independent pixel approximation (IPA) computations and additionally for an SZA of $60^{\circ}$ are summarised in Table II. The line marked 'original' in the table refers to a second computation performed on the original clouds and thus quantifies the sampling error of the MC radiative transfer calculations. For comparison: the mean reflectances of the stratocumulus clouds are $7.9 \%$ and $20.6 \%$ for an SZA of 0 or $60^{\circ}$, respectively. The same numbers for cumulus clouds are $4.5 \%$ and $9.6 \%$. The distribution of these values can be estimated from Figure 10.

Figure 8 shows that the coarse-grained fields suffer from considerable biases in the transmittance, which are reduced strongly by the downscaling algorithm. In Table II one can read that the biases are reduced by about an order of magnitude for the cumulus surrogates and a factor 3 to 4 for the stratocumulus surrogate fields. These factors are very similar for both the 3D MC and the IPA calculations.
In all cases the surrogates based on the exact spectrum are considerably more accurate. The surrogates without cloud fraction information reproduce the radiative fluxes more accurately than the coarse-grained fields, but are not as good as the standard surrogates. The improvements due to the increment adjustment are small, but statistically significant.

The RMSEs of the field mean transmittance and reflectance show a very similar picture, see Table III, except that for this measure the increment adjustment does not lead to improvements for the stratocumulus clouds and is even slightly detrimental for the cumulus clouds.

At the coarse pixel scale (Table IV) the reduction of the RMSE of the surrogate fields relative to the coarse-grained fields is smaller than at the scale of the full field. Again the extrapolation algorithm and the cloud fraction information are important. Whereas we did not find much improvement of the surrogates with respect to the high-resolution RMSE of the LWC, the reflectance still shows clear improvements at this scale (Table V).

The downscaling also improves the distribution of the radiative fluxes. This is exemplarily shown in Figure 9 for an SZA of $60^{\circ}$. In all cases (transmittance, reflectance and both SZAs) the distribution of the standard surrogate clouds is better than those of the coarse fields. The distribution of the surrogate fields match those of the original fields very 
Table III. RMS differences in mean reflectance and transmittance.

\begin{tabular}{|c|c|c|c|c|}
\hline \multirow[t]{2}{*}{ Field } & \multicolumn{2}{|c|}{ Cumulus } & \multicolumn{2}{|c|}{ Stratocumulus } \\
\hline & Refl. & Transm. & Refl. & Transm. \\
\hline \multicolumn{5}{|l|}{$S Z A=0^{\circ} 3 D R T$} \\
\hline Original & 0.0001 & 0.0001 & 0.0001 & 0.0001 \\
\hline Coarse field & 0.0258 & 0.0258 & 0.0103 & 0.0103 \\
\hline Extrapol. spectrum & 0.0024 & 0.0024 & 0.0029 & 0.0029 \\
\hline Exact spectrum & 0.0002 & 0.0002 & 0.0004 & 0.0004 \\
\hline No cloud cover & 0.0072 & 0.0072 & 0.0028 & 0.0028 \\
\hline No edge reduction & 0.0025 & 0.0025 & 0.0028 & 0.0028 \\
\hline \multicolumn{5}{|l|}{$S Z A=60^{\circ} 3 D R T$} \\
\hline Original & 0.0001 & 0.0001 & 0.0002 & 0.0002 \\
\hline Coarse field & 0.0491 & 0.0491 & 0.0352 & 0.0352 \\
\hline Extrapol. spectrum & 0.0055 & 0.0055 & 0.0125 & 0.0125 \\
\hline Exact spectrum & 0.0006 & 0.0006 & 0.0038 & 0.0038 \\
\hline No cloud cover & 0.0136 & 0.0136 & 0.0127 & 0.0127 \\
\hline No edge reduction & 0.0065 & 0.0065 & 0.0127 & 0.0127 \\
\hline \multicolumn{5}{|l|}{$S Z A=0^{\circ} I P A$} \\
\hline Original & 0.0001 & 0.0001 & 0.0001 & 0.0001 \\
\hline Coarse field & 0.0268 & 0.0268 & 0.0102 & 0.0102 \\
\hline Extrapol. spectrum & 0.0027 & 0.0027 & 0.0027 & 0.0027 \\
\hline Exact spectrum & 0.0001 & 0.0001 & 0.0004 & 0.0004 \\
\hline No cloud cover & 0.0075 & 0.0075 & 0.0029 & 0.0029 \\
\hline No edge reduction & 0.0031 & 0.0031 & 0.0027 & 0.0027 \\
\hline \multicolumn{5}{|l|}{$S Z A=60^{\circ} I P A$} \\
\hline Original & 0.0001 & 0.0001 & 0.0001 & 0.0001 \\
\hline Coarse field & 0.0484 & 0.0484 & 0.0351 & 0.0351 \\
\hline Extrapol. spectrum & 0.0049 & 0.0049 & 0.0128 & 0.0128 \\
\hline Exact spectrum & 0.0007 & 0.0007 & 0.0041 & 0.0041 \\
\hline No cloud cover & 0.0133 & 0.0133 & 0.0130 & 0.0130 \\
\hline No edge reduction & 0.0057 & 0.0057 & 0.0129 & 0.0129 \\
\hline
\end{tabular}

Table IV. Mean coarse-scale RMS differences in reflectance and transmittance.

\begin{tabular}{|c|c|c|c|c|}
\hline \multirow[t]{2}{*}{ Field } & \multicolumn{2}{|c|}{ Cumulus } & \multicolumn{2}{|c|}{ Stratocumulus } \\
\hline & Refl. & Transm. & Refl. & Transm. \\
\hline \multicolumn{5}{|l|}{$S Z A=0^{\circ} 3 D R T$} \\
\hline Original & 0.001 & 0.007 & 0.002 & 0.009 \\
\hline Coarse field & 0.026 & 0.127 & 0.012 & 0.030 \\
\hline Extrapol. spectrum & 0.003 & 0.032 & 0.005 & 0.018 \\
\hline Exact spectrum & 0.002 & 0.011 & 0.003 & 0.015 \\
\hline No cloud cover & 0.008 & 0.065 & 0.005 & 0.024 \\
\hline No edge reduction & 0.004 & 0.035 & 0.005 & 0.019 \\
\hline \multicolumn{5}{|l|}{$S Z A=60^{\circ} 3 D R T$} \\
\hline Original & 0.002 & 0.007 & 0.004 & 0.008 \\
\hline Coarse field & 0.049 & 0.163 & 0.039 & 0.074 \\
\hline Extrapol. spectrum & 0.007 & 0.065 & 0.016 & 0.042 \\
\hline Exact spectrum & 0.002 & 0.019 & 0.010 & 0.034 \\
\hline No cloud cover & 0.015 & 0.092 & 0.018 & 0.051 \\
\hline No edge reduction & 0.008 & 0.073 & 0.017 & 0.043 \\
\hline
\end{tabular}


Table V. High resolution RMS differences in reflectance and transmittance.

\begin{tabular}{lcccc}
\hline Field & \multicolumn{2}{c}{ Cumulus } & & \multicolumn{2}{c}{ Stratocumulus } \\
\cline { 2 - 3 } & Refl. & Transm. & Refl. & Transm. \\
\hline$S Z A=0^{\circ} 3 D R T$ & & & & 0.024 \\
Original & 0.006 & 0.028 & 0.034 & 0.086 \\
Coarse field & 0.027 & 0.212 & 0.032 & 0.182 \\
Extrapol. spectrum & 0.007 & 0.177 & 0.029 & 0.195 \\
Exact spectrum & 0.006 & 0.064 & 0.032 & 0.176 \\
No cloud cover & 0.010 & 0.177 & 0.032 & 0.193 \\
No edge reduction & 0.007 & 0.185 & & 0.196 \\
SZA =60 3D RT & & & 0.040 & 0.080 \\
Original & 0.008 & 0.027 & 0.068 & 0.243 \\
Coarse field & 0.051 & 0.256 & 0.057 & 0.260 \\
Extrapol. spectrum & 0.012 & 0.079 & 0.067 & 0.219 \\
Exact spectrum & 0.008 & 0.206 & 0.068 & 0.259 \\
No cloud cover & 0.018 & 0.212 & 0.263 \\
No edge reduction & 0.013 & &
\end{tabular}

(a)

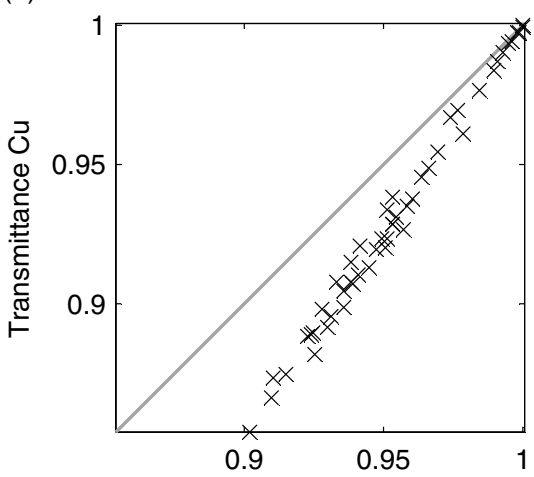

(c)

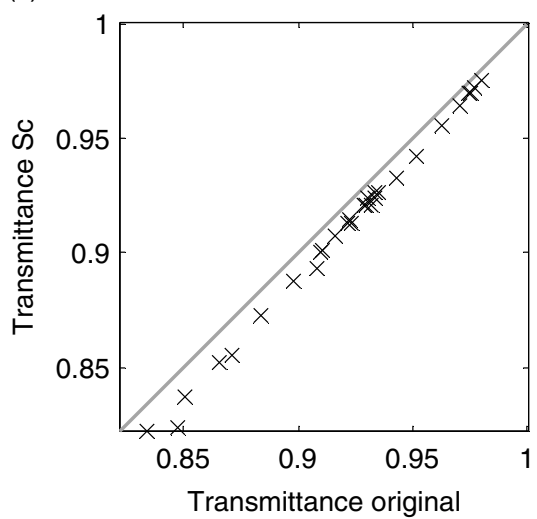

(b)

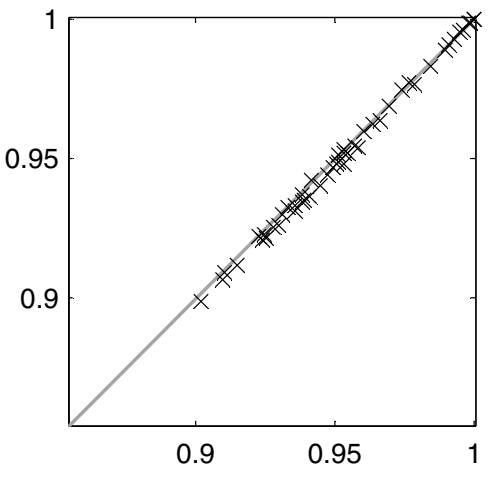

(d)

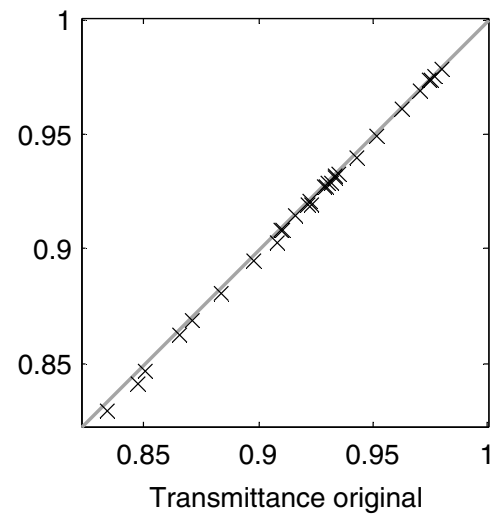

Figure 8. Scatterplot of the mean transmittance of the fields computed with a solar zenith angle of $0^{\circ}$. The top row ((a), (b)) contains the panels depicting the results for the cumulus clouds, the lower row ((c), (d)) for stratocumulus. The first column ((a), (c)) is for the coarse-grained original clouds, the right column ((b), (d)) for the standard surrogate clouds.

closely for cumulus and show a clear improvement over the coarse fields for the stratocumulus clouds.

Another comparison can be made on the anomalies, i.e. on the deviation between the high-resolution fluxes and their coarse-resolution averages. The distribution of these anomalies of the cumulus transmittance flux spans almost the full range from minus to plus unity, both in the original fields as well as in the surrogate fields. However, these anomalies of the coarse field range between \pm 0.2 and \pm 0.7 for SZAs of $0^{\circ}$ and $60^{\circ}$, respectively. Also in all other cases the downscaled surrogate fields match the anomaly distribution much closer than the coarse fields.

In all radiative flux fields, the coarse fields have a longer correlation length than the original clouds; the average autocovariance functions of the surrogate fields are always much closer to the average of the original fields.

A comparison of the radiative fluxes of the original LES clouds computed by $3 \mathrm{D}$ and IPA calculations shows that 

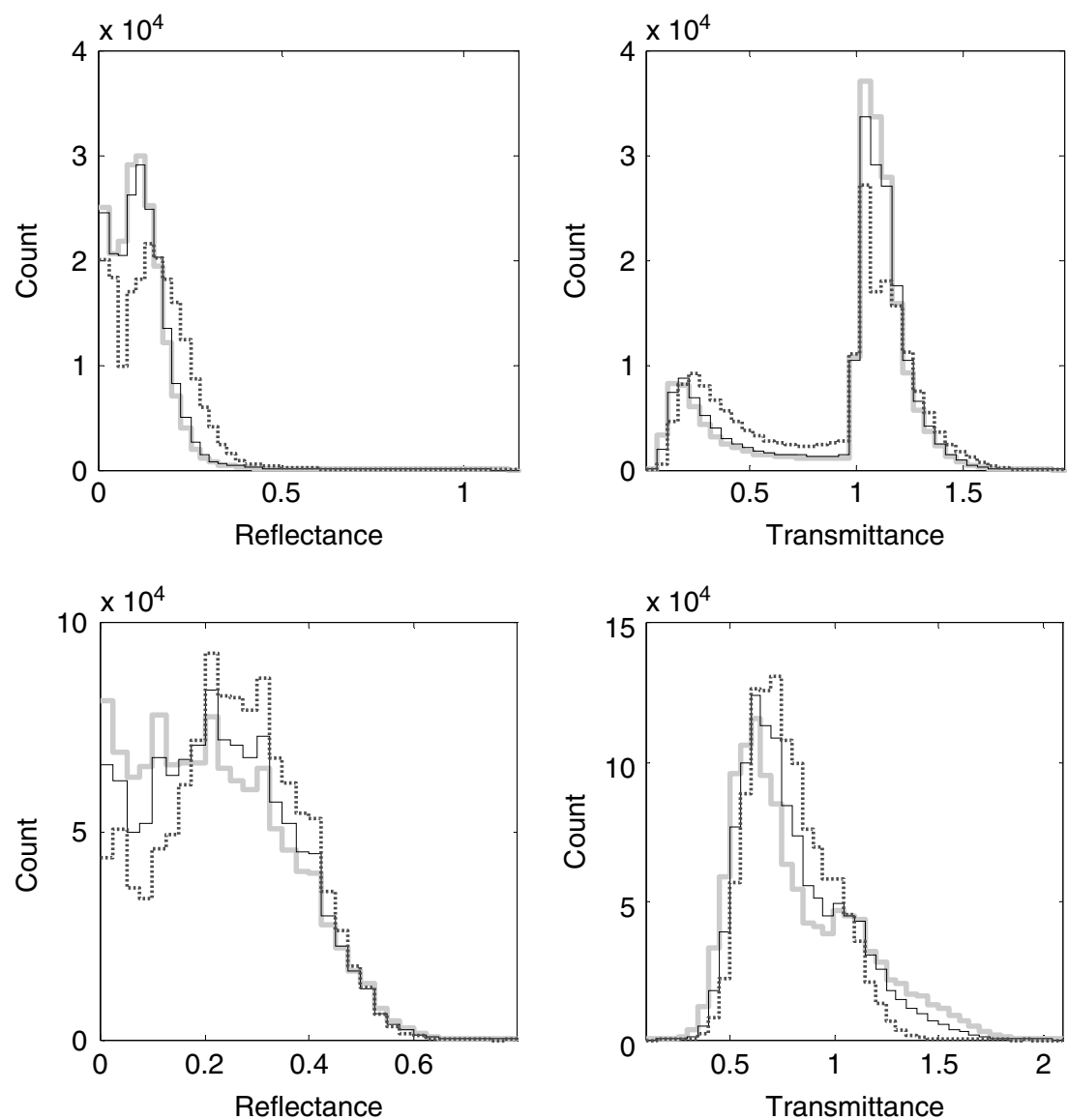

Figure 9. Histograms of the high-resolution reflectance and transmittance fluxes for an SZA of $60^{\circ}$. The thick grey line represents the distribution of the original LES clouds, the thin black line the standard surrogates with an extrapolated spectrum and the dotted line the coarse fields. The top row are the cumulus clouds, the lower row the stratocumulus clouds.

(a)

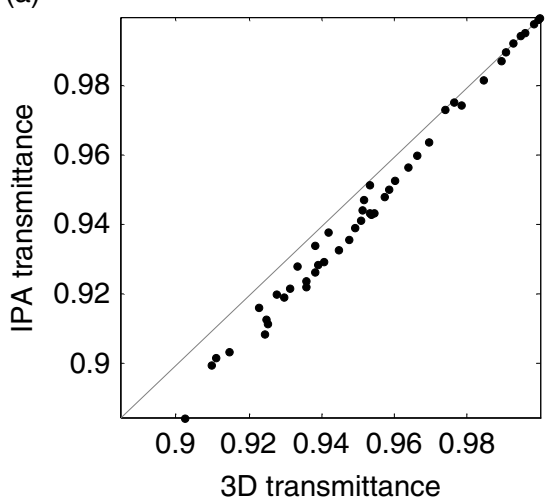

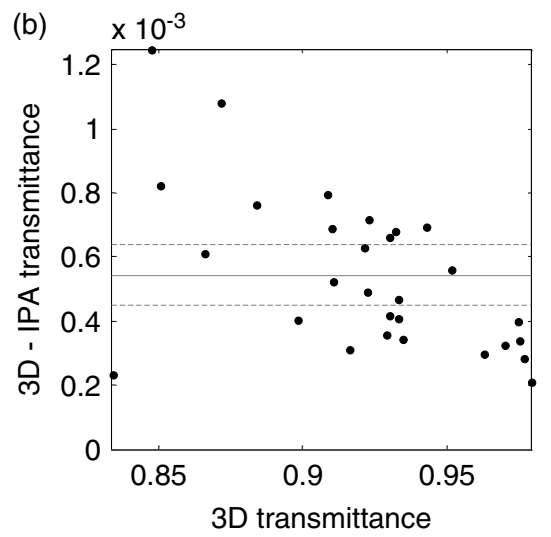

Figure 10. (a) Scatterplot of the transmittance of the cumulus clouds calculated with the full 3D MC model versus the radiative fluxes obtained with the independent pixel approximation (IPA). (b) Scatterplot of the 3D transmittance of the stratocumulus clouds versus the difference between the 3D and the IPA-computed transmittance. Both panels are calculated with a solar zenith angle of $0^{\circ}$. The drawn line indicates the mean difference and the dashed lines its two times sigma uncertainty.

these are statistically significantly different, i.e. so-called 3D radiative transfer effects due to the neglect of the horizontal photon transport are significant for these cloud fields (Figure 10). Thus getting the spatial correlations right can improve the optical properties of these cloud fields, especially for the cumulus fields. Still the difference between $3 \mathrm{D}$ and IPA is not large, thus for these fields the main improvements of the optical properties of the surrogate fields are likely due to improvements in the LWC distribution.

\section{Discussion}

At the original resolution, we found that the RMSE of the liquid water content and the optical depth is not improved by the downscaling. The surrogate fields can improve the RMSE by taking into account spatial correlations, e.g. if the coarse pixel to the north has a higher LWC, the largest sub-coarse pixel values will tend to be in the north as well. However, these improvements are offset by the subscale variability that is added, which increases the RMSE, but which is necessary to obtain unbiased radiative fluxes. The RMSE of the fluxes 
is improved at the original high resolution. Because the fluxes are determined by the optical cloud properties in a region around the high-resolution pixel, due to radiative smoothing (Marshak et al., 1998; Zinner et al., 2006), this situation is similar to analysing the errors at a coarser scale, which is discussed next.

For most applications, the results at coarser scales are most important. For example, before comparing the radiative transfer calculations performed on the high-resolution downscaled clouds, one would average them to the scale of a satellite measurement. At the resolution to which the LES clouds were coarsened, the optical depth of the surrogate cumulus clouds is almost a factor of 5 better than the optical depth of the coarse fields; the stratocumulus optical depth is a little better. These improvements are due to the nonlinear nature of the relation between liquid water content and optical depth. At this coarse resolution the radiative fluxes of the cumulus clouds are improved by a factor of 2.5 to 7 by the downscaling and about a factor 2 for the stratocumulus clouds. This is due to the nonlinear nature of the relationship between LWC and extinction (for a varying effective radius) and between optical depth and the transmission and reflection of light (e.g. King, 1987).

At the scale of the full cloud field, the bias and the RMSE of the optical depth and radiative fluxes of the cumulus surrogates is at least one order of magnitude smaller than the error of the coarse fields. The bias and RMSE of the optical depth and the fluxes of the stratocumulus clouds is a factor of 3 to 4 lower. Also, the distribution of the optical depth and the radiative fluxes is improved by downscaling the coarse fields. The correspondence between the distributions of the original fields and the surrogate fields indicate that these surrogate fields are better suited for studying 3D radiative transfer effects in cloudy atmospheres. Again we attribute the improvements to the nonlinear nature of the relation between LWC and optical depth and of radiative transfer itself, which is taken into account by the added small-scale variability.

One of the strengths of the downscaling algorithm is that it can handle any extrapolated spectrum and as a consequence any extrapolation algorithm. The accuracy of the results strongly depends on the quality achieved by the spectral extrapolation algorithm. To be able to study this, we have also generated surrogates with the Fourier spectrum of the original clouds fields. This spectrum represents the best possible spectrum an extrapolation method could compute. For stratocumulus clouds the extrapolation algorithm may approximate this upper limit well enough to obtain similar results. However, for the sparse cumulus clouds, this upper limit is likely not reachable: The Fourier spectrum of the original cloud contains information on the relative positions of the cloud clusters with respect to each other. For sparse cumulus clouds with many zero-valued pixels, this information is unambiguous enough to allow a very accurate reconstruction of the original cloud field; see also Venema et al. (2006a). Because the exact position of the clouds is a property of a certain realisation, but the positions will vary for every ensemble member, no extrapolation algorithm based on ensemble statistics will be able to estimate the spectrum with this accuracy. Thus for the cumulus clouds the results with the exact spectrum are only of theoretical interest.
However, it should be possible to develop better extrapolation algorithms than the one used for this study. The main limitation is that our extrapolation function is constant, whereas both cloud situations were nonstationary. In a similar non-stationary application, this could be remedied, e.g. by modelling the same transient situation with two models at two resolutions and using the highresolution model for the extrapolation (the coarse model could compute a larger ensemble or a larger region). The results for the surrogates based on the exact spectrum promise that with a better extrapolation algorithm an increase in quality similar to the current improvement from using coarse fields to using surrogates is possible. As the surrogates based on the exact spectrum still have a remaining error, we can conclude that the downscaling algorithm itself also contributes to the error.

In this study, the scale step was limited to a factor of four to ten. If larger steps are necessary, information on the distribution of the small-scale noise may become more important. However, the extrapolation algorithm for the power spectrum can only estimate the amount of subscale variance that needs to be added. The lack of information on the full distribution of the small-scale variability is likely the main reason for the remaining error in the surrogates with the exact Fourier spectrum of the original. More advanced downscaling algorithms may want to adjust the distribution of the subscale noise explicitly, based on information from higher-resolution models or measurements. This could be achieved by an additional iterative step that adjusts the subscale distribution, which would be similar to the adjustment of the full distribution in the standard IAAFT algorithm. Alternatively, the extrapolation of wavelet spectra (instead of or in addition to Fourier spectra) or structure functions may allow for a more dynamic estimation of the subscale variability on a case-by-case basis.

Information on the sub-coarse-scale cloud fraction is important. The version of the algorithm that does not use this information, however, still provides more accurate results than the coarse fields. For example, its radiative fluxes at the coarse scale are about a factor 2 more accurate. In applications where this information is not available, it is thus advisable to develop a parametrization for the cloud fraction. In a real application, the information on the cloud fraction will have an error itself. In this case, one may simultaneously perform the adjustment of the cloud fraction for multiple coarse pixels with about the same cloud fraction instead of one coarse pixel at a time. Combined with the spectral adjustment, this would allow for variations in cloud fraction based on information from the surrounding coarse pixels.

The adjustment of the distribution of the increments at the coarse pixel edges leads to a small improvement of the biases of the mean optical depth and radiative fluxes of the cumulus clouds, but can also lead to additional RMSE. For the stratocumulus clouds this adjustment does not have a significant influence on the error measures. Considering biases more important than RMSE, we decided to keep this adjustment. In preliminary surrogate clouds generated with a simpler extrapolation algorithm, the adjustment was visually judged to be needed and effective in reducing the large jumps at the edges. Thus for more difficult downscaling applications, this adjustment may be important. If the downscaling algorithm is applied to problems with a scale 
step larger than a factor ten, it may be useful to extend the edge adjustment to pairs of pixels further apart.

The suitability of our proposed downscaling algorithm for a particular application depends on the application's specific requirements. Whatever the application is, working with real high-quality high-resolution data is always preferred. Science should keep on striving to measure and model the atmosphere at higher resolutions; a downscaled field will always remain a surrogate solution, which is one reason to call these downscaled fields: surrogate fields. A second reason to view this algorithm as part of the surrogate line of research is that it is able to work with an arbitrary extrapolation function, in contrast to fractal methods, that impose a power law. We have a third reason to call the scaled-down fields: surrogate fields. Due to the structure of the algorithm, it is possible to combine this downscaling algorithm with the original algorithm we use for the stochastic generation of surrogate clouds (Venema et al., 2006a) and with upcoming similar algorithms. For example, surrogate clouds based on the LWC height distribution and spatial correlations from aircraft microphysics data, such as presented in Schmidt et al. (2007), can be combined with a coarse liquid water path field from satellite retrievals (Roebeling et al., 2008). The in situ information on the LWC height distribution will likely reduce the inaccuracies due to the fact that the current algorithm only estimates the subscale variance, but not the full subscale LWC distribution. The satellite would provide an overview and could deliver information on the temporal development of the mean liquid water content of the cloud field. These two algorithms are thus expected to complement each other very well.

\section{Conclusions and outlook}

This study presented a new downscaling method for threedimensional cloud fields. The algorithm was tested by coarse graining high-resolution 3D cloud fields from largeeddy simulations, scaling these fields down to the original fine resolution and analysing the difference between the original LES cloud fields and the downscaled surrogate cloud fields.

A downscaling algorithm is needed because of the nonlinear relationship between liquid water content and optical depth and the nonlinear nature of the radiative transfer equations. The algorithm avoids systematic errors by adding small-scale variability. Our algorithms include a first attempt to do so for radiative transfer through 3D cloud fields with known subscale cloud fraction.

The differences were analysed with respect to four error measures: the mean root-mean-square error at the original resolution of the LES clouds, the mean RMSE at the coarse resolution, and the RMSE of the mean field properties, as well as the bias in the mean field properties. The improvement achieved by the downscaling is larger the larger the scales considered, which is fortunate because most applications will be interested at the coarser scales. For example, at the largest scale (the full cloud field), the bias and the RMSE of the optical depth and radiative fluxes of the cumulus surrogates is at least one order of magnitude smaller than the error of the coarse fields. The bias and RMSE of the optical depth and the fluxes of the stratocumulus clouds are a factor of 3 to 4 lower. The optical depth and radiative flux distributions are also improved by downscaling the coarse fields.
Further improvements can be achieved by introducing more-advanced extrapolation methods for the power spectrum of the coarse fields. The current algorithm only estimates the variance missing at sub-coarse pixel scales; future versions could include a more accurate description of the subscale distribution.

A very promising prospect is a combination of this downscaling algorithm with the standard algorithm to generate surrogate clouds. For example, coarse 2D satellite imager fields could then be combined with high-resolution in situ data or observations from active cloud remotesensing instruments to generate $3 \mathrm{D}$ cloud fields. The imager would provide the overview and could give information on the temporal development, whereas the high-resolution distribution would improve the accuracy of the cloud products.

\section{Acknowledgements}

We would like to thank Hartwig Deneke very much for valuable suggestions on the analysis of the results and the presentation of this study. This research was started as part of the $4 \mathrm{D}$-clouds project, which was funded by the German Ministry of Research, BMBF, in the AFO2000 Program on Atmospheric Research. For finalising this work, we gratefully acknowledge financial support by the SFB/TR 32 'Pattern in Soil-Vegetation-Atmosphere Systems: Monitoring, Modelling, and Data Assimilation' and the research project on surrogate clouds, grant VE366/3-1, both funded by the Deutsche Forschungsgemeinschaft (DFG).

\section{References}

Basu S, Foufoula-Georgiou E, Porté-Agel F. 2004. Synthetic turbulence, fractal interpolation, and large-eddy simulation. Phys. Rev. E 70: 026310, DOI:10.1103/PhysRevE.70.026310.

Brenguier J-L, Pawlowska H, Schüller L, Preusker R, Fischer J, Fouquart Y. 2000. Radiative properties of boundary layer clouds: Droplet effective radius versus number concentration. J. Atmos. Sci. 57: $803-821$.

Brown AR, Cederwall RT, Chlond A, Duynkerke PG, Golaz J-C, Khairoutdinov M, Lewellen DC, Lock AP, MacVean MK, Moeng C-H, Neggers RAJ, Siebesma AP, Stevens B. 2002. Large-eddy simulation of the diurnal cycle of shallow cumulus convection over land. Q. J. R. Meteorol. Soc. 128: 1075-1093.

Cahalan RF, Ridgway W, Wiscombe WJ, Bell TL, Snider JB. 1994. The albedo of fractal stratocumulus clouds. J. Atmos. Sci. 51: 2434-2455.

Chambers LH, Wielicki BA, Evans KF. 1997. Accuracy of the independent pixel approximation for satellite estimates of oceanic boundary layer cloud optical depth. J. Geophys. Res. 102: 1779-1794.

Chosson F, Brenguier J-L, Schüller L. 2007. Entrainment-mixing and radiative transfer simulation in boundary layer clouds. J. Atmos. Sci. 64: $2670-2682$.

Davis A, Marshak A, Cahalan R, Wiscombe W. 1997. The Landsat scale break in stratocumulus as a three-dimensional radiative transfer effect: Implications for cloud remote sensing. J. Atmos. Sci. 54: 241-260.

Davis AB, Marshak A, Gerber H, Wiscombe WJ. 1999. Horizontal structure of marine boundary layer clouds from centimeter to kilometer scales. J. Geophys. Res. 104: 6123-6144.

Erkelens JS, Venema VKC, Russchenberg HWJ, Ligthart LP. 2001. Coherent scattering of microwaves by particles: Evidence from clouds and smoke. J. Atmos. Sci. 58: 1091-1102.

Ferraris L, Gabellani S, Rebora N, Provenzale A. 2003. A comparison of stochastic models for spatial rainfall downscaling. Water Resour. Res. 39: 1368, DOI:10.1029/2003WR002504.

Gimeno García S, Trautmann T. 2003. 'Radiative transfer modeling in inhomogeneous clouds by means of the Monte Carlo method'. Wissenschaftliche Mitteilungen aus dem Institut für Meteorologie der Universität Leipzig, No. 30, Meteorological Institute of Leipzig University: Germany. 
Hogan RJ, Kew SF. 2005. A 3D stochastic cloud model for investigating the radiative properties of inhomogeneous cirrus clouds. Q. J. R. Meteorol. Soc. 131: 2585-2608.

King MD. 1987. Determination of the scaled optical thickness of clouds from reflected solar radiation measurements. J. Atmos. Sci. 44: 1734-1751.

Koren I, Oreopoulos L, Feingold G, Remer LA, Altaratz O. 2008. How small is a small cloud? Atmos. Chem. Phys. 8: 3855-3864.

Marani M, Zanetti S. 2007. Downscaling rainfall temporal variability. Water Resour. Res. 43: W09415, DOI:10.1029/2006WR005505.

Marshak A, Davis A, Cahalan RF, Wiscombe W. 1998. Nonlocal independent pixel approximation: Direct and inverse problems. IEEE Trans. Geosci. Remote Sensing 36: 192-205.

Olsson J. 1998. Evaluation of a scaling cascade model for temporal rainfall disaggregation. Hydrol. Earth Sys. Sci. 2: 19-30.

Peng YR, Lohmann U. 2003. Sensitivity study of the spectral dispersion of the cloud droplet size distribution on the indirect aerosol effect. Geophys. Res. Lett. 30: 1507, DOI:10.1029/2003GL017192

Perica S, Foufoula-Georgiou E. 1996. Model for multiscale disaggregation of spatial rainfall based on coupling meteorological and scaling descriptions. J. Geophys. Res. 101: 26347-26361.

Roebeling RA, Deneke HM, Feijt AJ. 2008. Validation of cloud liquid water path retrievals from SEVIRI using one year of CloudNET observations. J. Appl. Meteorol. Clim. 47: 206-222.
Schmidt KS, Venema V, Di Giuseppe F, Scheirer R, Wendisch M, Pilewskie P. 2007. Reproducing cloud microphysical and irradiance measurements using three 3D cloud generators. Q. J. R. Meteorol. Soc. 133: 765-780.

Sheng J, Zwiers F. 1998. An improved scheme for time-dependent boundary conditions in atmospheric general circulation models. Clim. Dyn. 14: 609-613.

Slingo A. 1989. A GCM parameterization for the shortwave radiative properties of water clouds. J. Atmos. Sci. 46: 1419-1427.

Tatarski VI. 1961. Wave propagation in a turbulent medium. McGrawHill: New York.

Venema V, Meyer S, Gimeno García S, Kniffka A, Simmer C, Crewell S, Löhnert U, Trautmann T, Macke A. 2006a. Surrogate cloud fields generated with the Iterative Amplitude Adapted Fourier Transform algorithm. Tellus 58A: 104-120.

Venema V, Ament F, Simmer C. 2006b. A Stochastic Iterative Amplitude Adjusted Fourier Transform algorithm with improved accuracy. Nonlinear Processes in Geophys. 13: 321-328.

Venema V, Bachner S, Rust HW, Simmer C. 2006c. Statistical characteristics of surrogate data based on geophysical measurements. Nonlinear Processes in Geophys. 13: 449-466.

Zinner T, Mayer B, Schröder M. 2006. Determination of threedimensional cloud structures from high-resolution radiance data. J. Geophys. Res. 111: D08204, DOI:10.1029/2005JD006062. 